\title{
Identification of an Acidic Amino Acid Permease Involved in D-Aspartate Uptake in the Yeast Cryptococcus humicola
}

\author{
Daiki Imanishi, Yoshio Kera and Shouji Takahashi *
}

Citation: Imanishi, D.; Kera, Y.; Takahashi, S. Identification of an Acidic Amino Acid Permease Involved in D-Aspartate Uptake in the Yeast Cryptococcus humicola. Microorganisms 2021, 9, 192. https:// doi.org/10.3390/microorganisms 9010192

Academic Editor: Peter Richard

Received: 14 December 2020

Accepted: 14 January 2021

Published: 18 January 2021

Publisher's Note: MDPI stays neutral with regard to jurisdictional claims in published maps and institutional affiliations.

Copyright: (c) 2021 by the authors. Licensee MDPI, Basel, Switzerland. This article is an open access article distributed under the terms and conditions of the Creative Commons Attribution (CC BY) license (https:/ / creativecommons.org/licenses/by/ $4.0 /)$.
Department of Bioengineering, Nagaoka University of Technology, Nagaoka, Niigata 940-2188, Japan; s175037@stn.nagaokaut.ac.jp (D.I.); yoshkera@nagaokaut.ac.jp (Y.K.)

* Correspondence: shoutaka@vos.nagaokaut.ac.jp; Tel.: +81-258-47-9667

Abstract: D-aspartate oxidase (DDO) catalyzes the oxidative deamination of acidic D-amino acids, and its production is induced by D-Asp in several eukaryotes. The yeast Cryptococcus humicola strain UJ1 produces large amounts of DDO (ChDDO) only in the presence of D-Asp. In this study, we analyzed the relationship between D-Asp uptake by an amino acid permease (Aap) and the inducible expression of ChDDO. We identified two acidic Aap homologs, named "ChAap4 and ChAap5," in the yeast genome sequence. ChAAP4 deletion resulted in partial growth defects on D-Asp as well as L-Asp, L-Glu, and L-Phe at $\mathrm{pH} 7$, whereas ChAAP5 deletion caused partial growth defects on L-Phe and L-Lys, suggesting that ChAap4 might participate in D-Asp uptake as an acidic Aap. Interestingly, the growth of the Chaap4 strain on D- or L-Asp was completely abolished at pH 10, suggesting that ChAap4 is the only Aap responsible for D- and L-Asp uptake under high alkaline conditions. In addition, ChAAP4 deletion significantly decreased the induction of DDO activity and ChDDO transcription in the presence of D-Asp. This study revealed that D-Asp uptake by ChAap4 might be involved in the induction of ChDDO expression by D-Asp.

Keywords: D-aspartate oxidase; amino acid permease; Cryptococcus humicola; D-aspartate; gene expression

\section{Introduction}

D-aspartate oxidase (DDO or DASPO, EC 1.4.3.1) is a peroxisomal enzyme that catalyzes the oxidative deamination of acidic D-amino acids to produce their corresponding $\alpha$-keto acids and ammonia. DDO has been found in various eukaryotic organisms, ranging from fungi to mammals, but it is not present in prokaryotic organisms [1-5]. To date, DDO genes have been cloned from various eukaryotic organisms including human and mouse, and their enzymatic properties have been extensively studied [6]. DDO of the yeast C. humicola strain UJ1 (ChDDO) is the first DDO cloned from a microorganism and has high substrate specificity and high activity for D-Asp [5,7]. In fungi, DDO participates in the assimilation and detoxification of acidic D-amino acids [8]. In animals, the enzyme is considered to regulate D-Asp levels in relation to some important physiological functions, such as hormone secretion and neurotransmission, and suggested to associate with the psychiatric disorder schizophrenia in human [9-11]. Therefore, it might be important to reveal the environmental and protein factors that can affect the expression of DDO gene.

Yeast cells can grow on various L- and D-amino acids as carbon, nitrogen, or both sources $[8,12,13]$. Amino acids are intracellularly catabolized by various enzymes, such as oxidase, deaminase, transaminase, to yield nitrogen in the form of ammonium, glutamate, or glutamine as nitrogen donors in biosynthetic reactions [13]. On the other hand, the carbonaceous products from the catabolic reactions enter central metabolic pathways including tricarboxylic acid cycle and gluconeogenesis pathway to be used as carbon sources in biosynthetic reactions or energy source [13].

Cryptococcus humicola strain UJ1 (recently reclassified as Vanrija humicola strain UJ1) is a basidiomycetous yeast that can utilize D-Asp as a sole source of carbon, nitrogen, 
or both and expresses significant levels of ChDDO only in the presence of D-Asp in culture medium $[5,8]$. This inducible expression of ChDDO in response to D-Asp is regulated at the transcriptional level [8]. This acidic D-amino acid-dependent induction of DDO activity has also been reported in other organisms. For example, DDO activity in the fungus Fusarium sacchari var. elongatum strain Y-105 increases when it is grown on D-Asp or D-Glu as the sole source of carbon and nitrogen [14]. DDO activity in the yeast Candida boidinii strain 2201 increases when it is grown on D-Glu as the sole source of nitrogen [15]. The oral or intraperitoneal administration of D-Asp to mice increases DDO activity in the liver and kidneys [16]. The oral administration of D-Asp to pregnant rats increases DDO activity in the livers and kidneys of newborn rats [9]. These findings suggest that the inducible expression of DDO in the presence of acidic D-amino acids might be widely distributed in eukaryotic organisms. However, the induction mechanism remains to be elucidated.

In C. humicola UJ1, the induction of ChDDO expression by D-Asp is significantly repressed by the copresence of L-Asp in media [5,8]. This repression is considered to be caused by the decreased uptake of D-Asp via competitive inhibition by L-Asp. In fungi, amino acids are imported into cells by amino acid permeases (Aaps) located in the plasma membrane. Most yeast Aaps belong to the yeast amino acid transporter (YAT) family, a group of proteins in the amino acid-polyamine-organocation (APC) superfamily [17]. Aap homologs of the APC superfamily are found in fungi as well as animals, plants, and bacteria [18]. The typical YAT possesses 12 transmembrane regions (TMs) and functions as a symporter through proton-driven secondary active transport $[19,20]$. In the yeast Saccharomyces cerevisiae, 19 Aaps have been identified [17]. The yeast Aaps have different substrate specificities. Most yeast Aaps have high affinity and specificity for specific amino acids, whereas the general Aap Gap1p is involved in the uptake of all naturally occurring Lamino acids as well as some D-amino acids and amino acids analogs [20-23]. In S. cerevisiae, acidic L-amino acids are imported by the dicarboxylic amino acid transporter Dip5p, as well as Gap1p [24]. Dip5p mediates the high affinity and transport-capacity transport of L-Asp and L-Glu [24,25]. The Aaps specific for dicaboxylic amino acids have been also identified and characterized in Aspergillus nidulans (AgtA) and Penicillium chrysogenum (PcDip5) [26,27]. However, it is unclear whether dicarboxylic Aaps can also mediate the uptake of acidic D-amino acids and whether they are involved in the induction of DDO expression by D-Asp in yeast. In addition, the reason why multiple Aaps contribute to acidic amino acid uptake is unclear.

In this study, as the first step to elucidate the mechanism of ChDDO induction by D-Asp in C. humicola UJ1, we investigated the relationship between D-Asp uptake by a dicarboxylic Aap and the D-Asp-induced expression of ChDDO. To reveal the relationship, we identified an acidic Aap, ChAap4, and analyzed the expression of ChDDO in a ChAAP4disrupted strain. Our results revealed that ChAap4 might participate in D-Asp uptake into cells and, consequently, indirectly the inducible expression of ChDDO, and the protein is essential for growth on D-Asp and L-Asp at a high alkaline $\mathrm{pH}$, illustrating the involvement of D-Asp uptake by acidic Aap in the inducible expression of ChDDO in the presence of D-Asp.

\section{Materials and Methods}

\subsection{Materials}

Yeast nitrogen base without amino acids (YNB), yeast carbon base (YCB), and YNB without amino acids and ammonium sulfate (YNB w/o AA and AS) were obtained from Difco (Detroit, MI, USA). D-Asp was a generous gift from Mitsubishi Tanabe Pharmaceutical (Osaka, Japan). Other amino acids were procured from Nacalai Tesque (Kyoto, Japan). All other chemicals were purchased from Wako Pure Chemical Industries (Osaka, Japan), Nacalai Tesque, or Sigma-Aldrich (St Louis, MO, USA). DNA polymerase was purchased from Takara Bio (Shiga, Japan). PCR primers (Table S1) were synthesized by Eurofins Genomics (Tokyo, Japan). 


\subsection{Strains, Media, and Growth Conditions}

C. humicola strain UJ1 [5] was used as the wild-type strain. C. humicola strain UM3 [28], a ura3 mutant derived from strain UJ1, was used as the host for gene disruption experiments. The yeast cells were routinely grown in YPD medium $(1 \%(w / v)$ yeast extract, $2 \%(w / v)$ peptone, $2 \%(w / v)$ glucose), or SD medium $(0.67 \%(w / v) \mathrm{YNB}, 2 \%(w / v)$ glucose). Wherever required, $20 \mu \mathrm{g} / \mathrm{mL}$ uracil was added to SD medium. The growth test of ChAAP-disrupted strains (Chaap) was performed as follows. Cells were precultured in SD medium at $30^{\circ} \mathrm{C}$ for $16 \mathrm{~h}$. The preculture was inoculated into synthetic medium containing $1.17 \%(w / v)$ YCB with $10 \mathrm{mM}$ of each nitrogen source at a cell density of $\mathrm{OD}_{600}=0.005$. The initial $\mathrm{pH}$ was adjusted to 7.0. For solid medium, $2 \%(w / v)$ agar was added to the culture medium. Escherichia coli strain DH5 $\alpha$ was employed as a host for propagating plasmids. E. coli cells were grown at $37^{\circ} \mathrm{C}$ in LB medium $(1 \%(w / v)$ tryptone, $0.5 \%(w / v)$ yeast extract, $0.5 \%$ $(w / v) \mathrm{NaCl})$ supplemented with $100 \mu \mathrm{g} / \mathrm{mL}$ ampicillin.

\subsection{Identification of Aap Homologs}

The putative Aaps of strain UJ1 were identified in the yeast draft genome sequence data [29] via a BlastP search [30] using the amino acid sequences of $C$. neoformans Aaps 1-8 [31]. A phylogenetic tree based on the amino acid sequences of Aaps was constructed by the neighbor-joining method using Molecular Evolutionary Genetics Analysis version 7 [32]

\subsection{DNA and RNA Preparation}

The total DNA of the yeast was prepared as described previously [28]. To prepare total RNA, cells were washed twice with ice-cold water, resuspended in ice-cold water, and then transferred to a $2 \mathrm{~mL}$ screw tube containing an equal volume of $\varphi 0.45-0.5 \mathrm{~mm}$ zirconia beads (BioSpec Products, Inc., Bartlesville, OK, USA). After centrifugation at $12,000 \times g$ for $2 \mathrm{~min}$ at $4{ }^{\circ} \mathrm{C}$, the supernatant was removed, the cells were lyophilized using a freeze dryer system (DRC-1100 and FDU-2100, EYELA, Tokyo, Japan), and stored at $-80{ }^{\circ} \mathrm{C}$ until use. The tube containing the lyophilized cells was shaken vigorously for 5 min using a vortex mixer. Total RNA was extracted and purified from the disrupted cells using a Direct-zol RNA Miniprep Kit (ZYMO Research, Irvine, CA, USA) according to the manufacturer's instructions.

\subsection{Disruption of ChAAP Genes}

An approximately $2.0 \mathrm{kbp}$ ChURA3 fragment was amplified using Tks Gflex DNA polymerase (Takara Bio) with the primer set URA3F/URA3R (Table S1) and the yeast vector pICUG as a template [28]. Approximately, $1.0 \mathrm{~kb}$ of the $5^{\prime}$ - and $3^{\prime}$-regions of ChAAP4 and ChAAP5 was amplified using the following primer sets: AAP4UF/AAP4UR for the ChAAP4 5'-region, AAP4DF/AAP4DR for the ChAAP4 3'-region, AAP5UF/AAP5UR for the ChAAP5 5'-region, and AAP5DF/AAP5DR for the ChAAP5 $3^{\prime}$-region (Table S1). Yeast genome DNA was employed as a template. The amplified ChURA3 and 5'- and $3^{\prime}$-fragments of each gene were mixed, and the fused DNA fragments were obtained via overlap extension PCR using the $5^{\prime}$ - and $3^{\prime}$-terminal primers for each gene. The resulting disruption cassette for each ChAAP gene (approximately, $5.0 \mathrm{kbp}$ ) was introduced into the yeast cells via electroporation using a MicroPulser Electroporator (Bio-Rad, Hercules, CA, USA) as described previously [28]. Transformants were selected for their ability to grow on SD medium without uracil at $30{ }^{\circ} \mathrm{C}$ for 3 days. The disruption of ChAAP4 and ChAAP5 was confirmed by PCR using the primer sets $4 \mathrm{UFwd} 1 / 4 \mathrm{URev} 1$ and $4 \mathrm{DFwd} 2 / 4 \mathrm{DRev} 2$ for Chaap 4 and 5UFwd2/5URev2 and 5DFwd3/5DRev3 for Chaap5 (Table S1). In these gene disruption mutants, ChURA3 was inserted, in the opposite direction, into the genomic ChAAP4 between 117 and $309 \mathrm{bp}$ and ChAAP5 between 716 and $1368 \mathrm{bp}$ (Figure 1). 


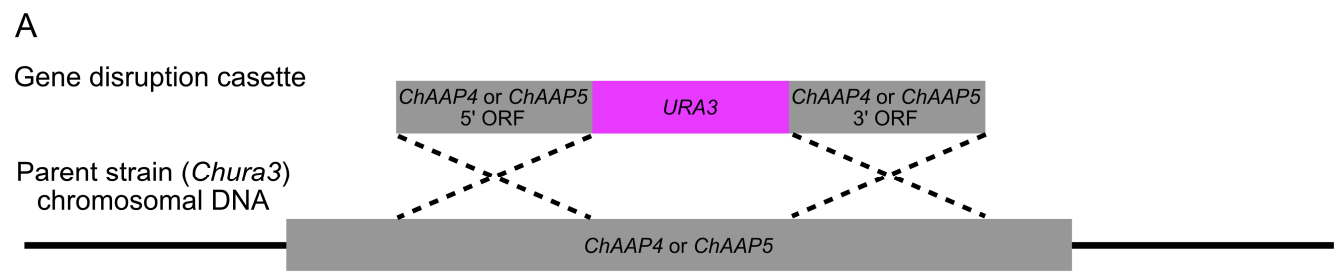

B
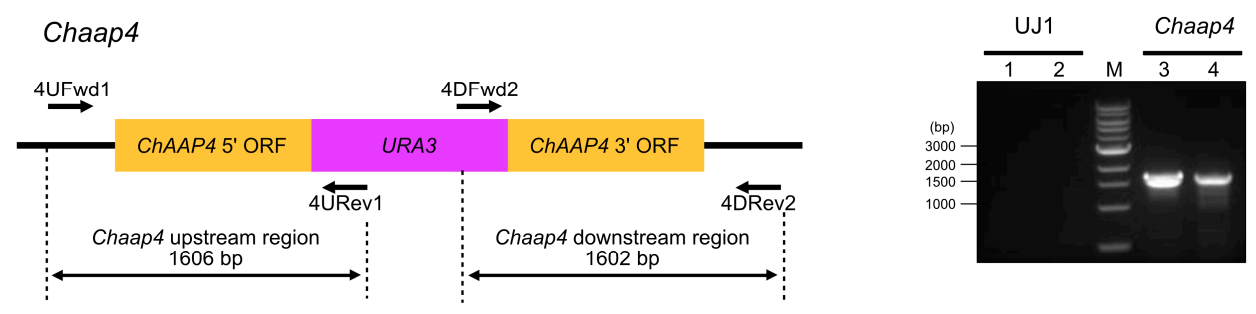

C
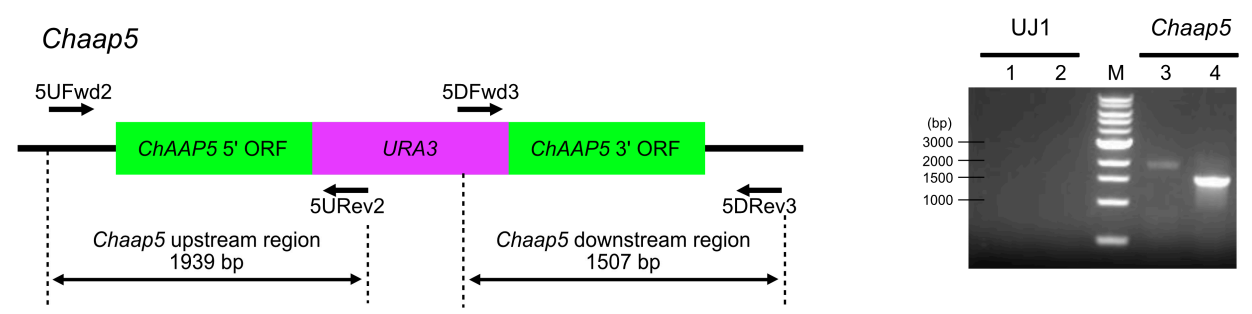

Figure 1. Construction of Chaap4 and Chaap5 strains. (A) Schematic representation of ChAAP4 and ChAAP5 gene disruptions by homologous recombination using ChURA3. (B) PCR analysis of ChAAP4 gene disruption. The numbers above the box indicate the distance (bp) from the start codon ATG of the chromosomal ChAAP4. ChURA3 was inserted, in the opposite direction, into the ChAAP4 between 117 and $309 \mathrm{bp}$. Lanes 1 and 2, the negative control amplification of the upstream and the downstream regions, respectively, in the wild-type strain (strain UJ1); lanes 3 and 4, the amplification of the upstream and downstream regions in Chaap4 strain. (C) PCR analysis of ChAAP5 gene disruption. The numbers above the box indicate the distance (bp) from the start codon ATG of the chromosomal ChAAP5. ChURA3 was inserted, in the opposite direction, into the genomic ChAAP5 between 716 and $1368 \mathrm{bp}$. Lanes 1 and 2, the negative control amplification of the upstream and the downstream regions, respectively, in the wild-type strain (strain UJ1); lanes 3 and 4, the amplification of the upstream and the downstream regions in Chaap5 strain. PCR was performed using Chaap4- or Chaap5-specific primer pairs in Table S1.

\subsection{ChDDO Induction Experiment}

Cells were precultured in $\mathrm{SD}$ medium at $30^{\circ} \mathrm{C}$ for $16 \mathrm{~h}$. The preculture was inoculated into fresh SD medium at a final cell density of $\mathrm{OD}_{600}=0.05$ and further grown at $30^{\circ} \mathrm{C}$ for $16 \mathrm{~h}$ with shaking at $166 \mathrm{rpm}$. The cells were collected by centrifugation and washed twice with ice-cold water, and $50 \mathrm{OD}_{600}$ units of cells were resuspended in synthetic medium (YNB w/o AA and AS) or containing $60 \mathrm{mM}$ D-Asp for each $\mathrm{pH}$ condition. After incubation at $30^{\circ} \mathrm{C}$ for $3 \mathrm{~h}$ with shaking, the cells were harvested by centrifugation and washed twice with ice-cold water.

\subsection{DDO Assay}

DDO activity was measured as described by Takahashi et al. [8]. Briefly, cells were washed twice with ice-cold lysis buffer ( $50 \mathrm{mM}$ potassium phosphate, $\mathrm{pH}$ 8.0, $2 \mathrm{mM}$ EDTA), resuspended in the same buffer, and then transferred to a $2 \mathrm{~mL}$ screw tube containing an equal volume of $\varphi 0.45-0.5 \mathrm{~mm}$ glass beads. The tube was shaken vigorously for $1 \mathrm{~min}$ using a Mini Bead Beater-8 (BioSpec Products), followed by cooling on ice for $3 \mathrm{~min}$. This procedure was repeated eight times. The extract was clarified via centrifugation at $20,000 \times g$ for $30 \mathrm{~min}$ at $4{ }^{\circ} \mathrm{C}$, and the supernatant was used as the crude cell extract for the enzyme assay. The enzymatic activity was determined spectrophotometrically via 
a horseradish peroxidase (HRP)-coupled reaction with phenol and 4-aminoantipyrine (4-AA) [33]. The reaction mixture contained $20 \mathrm{mM}$ D-Asp, $20 \mu \mathrm{M}$ FAD, $2 \mathrm{mM}$ phenol, $1.5 \mathrm{mM} 4-\mathrm{AA}$, and $2.5 \mathrm{U} / \mathrm{mL}$ HRP (Sigma-Aldrich) in $50 \mathrm{mM}$ potassium phosphate buffer ( $\mathrm{pH}$ 8.0). The enzymatic activity was determined at $505 \mathrm{~nm}$ using a molar extinction coefficient for a generated quinone imine of $6580 \mathrm{M}^{-1} \mathrm{~cm}^{-1}$.

\subsection{Quantitative Real-Time RT-PCR ( $q R T-P C R$ )}

Total RNA was isolated from cells grown in synthetic medium (YNB w/o AA and AS) containing $60 \mathrm{mM}$ D-Asp. qRT-PCR of ChDDO transcripts was performed using RNAdirect SYBR Green Realtime PCR Master Mix (Toyobo, Osaka, Japan) with the primer set RTChDDOF2/RTChDDOR2 (Table S1) in a StepOne real-time PCR system (Applied Biosystems, Foster City, CA, USA). Transcription of TAF10 was determined as a normalizing gene using the primer set RTChTAF10F/RTChTAF10R, and the relative transcriptional levels of $C h D D O$ were calculated against the normalizing gene using the $2^{-\Delta \Delta \mathrm{Ct}}$ method [34].

\subsection{Sequence Analyses and Structural Modeling}

An amino acid sequence alignment was created using T-coffee (http:/ / tcoffee.crg.cat/). Sequence comparisons were performed using EMBOSS Needle Pairwise Sequence Alignment (https://www.ebi.ac.uk/Tools/psa/emboss_needle/). Three-dimensional structure models were generated using I-TASSER (http://zhanglab.ccmb.med.umich.edu/) and visualized using PyMOL ver. 2.3 (http:/ / www.pymol.org/).

\section{Results}

\subsection{Identification of Acidic Aap Homologs of C. humicola Strain UJ1}

To identify the Aap homologs of C. humicola strain UJ1, we searched for homologs in the draft genome sequence using the amino acid sequences of Aaps 1-8 of $C$. neoformans [31]. A BLASTP search identified 31 Aap homologs of strain UJ1 with significant similarities (E-value $<1.0 \times 10^{-50}$ and amino acid sequence identity $>25 \%$ ) to C. neoformans Aaps. It has been reported that the YATs responsible for the transport of similar amino acids (proline, acidic amino acids, basic amino acids, branched-chain amino acids, aromatic amino acids, and various amino acids) are classified into the same clade of a phylogenetic tree [17]. To predict which of the 31 Aap homologs of strain UJ1 is the acidic Aap, we constructed a phylogenetic tree using their amino acid sequences and those of known YATs (Figure 2). The phylogenetic tree illustrated that two Aap homologs, named "ChAap4 (g920) and ChAap5 (g5887)," belong to the same clade as acidic YATs (CnAap4, CnAap5, Dip5p, and AgtA).

ChAap 4 and ChAap 5 contained 573 and 540 amino acid residues, respectively, and they exhibited the highest amino acid sequence identities with CnAap4 $(69.2 \%$ and $63.5 \%$, respectively). In addition, ChAap4 displayed $41.5 \%, 41.7 \%$, and $43.9 \%$ amino acid identities with Dip5p, PcDip5, and AgtA, respectively, and ChAap5 exhibited 40.1\%, 38.9\%, and $41.2 \%$ amino acid identities, respectively, with those same enzymes. Both C. humicola acidic Aap homologs were suggested to have $12 \mathrm{TM}$ regions, and their $\mathrm{N}$-terminal and C-terminal regions were predicted to be located in the cytoplasm, matching the typical topologies of YATs (Figure S1). In addition, the positions of the TM regions were mostly consistent with those of the E. coli arginine transporter AdiC. The GXG (where $X$ is any amino acid) and $(\mathrm{F} / \mathrm{Y})(\mathrm{S} / \mathrm{A} / \mathrm{T})(\mathrm{F} / \mathrm{Y}) \mathrm{XGXE}$ motifs were found in TM1 and TM6, respectively (Figure 3), and these motifs are highly conserved in Aaps and involved in the interactions with the $\alpha$ carboxy and $\alpha$-amino groups of amino acid substrates, respectively $[17,35]$. These findings suggested that ChAap4 and ChAap5 might function as acidic Aaps in strain UJ1. However, some amino acid residues conserved in dicarboxylic Aaps (AgtA, Dig5p, and PcDip5) were not conserved in C. humicola Aaps (Figure S1) [27]. 


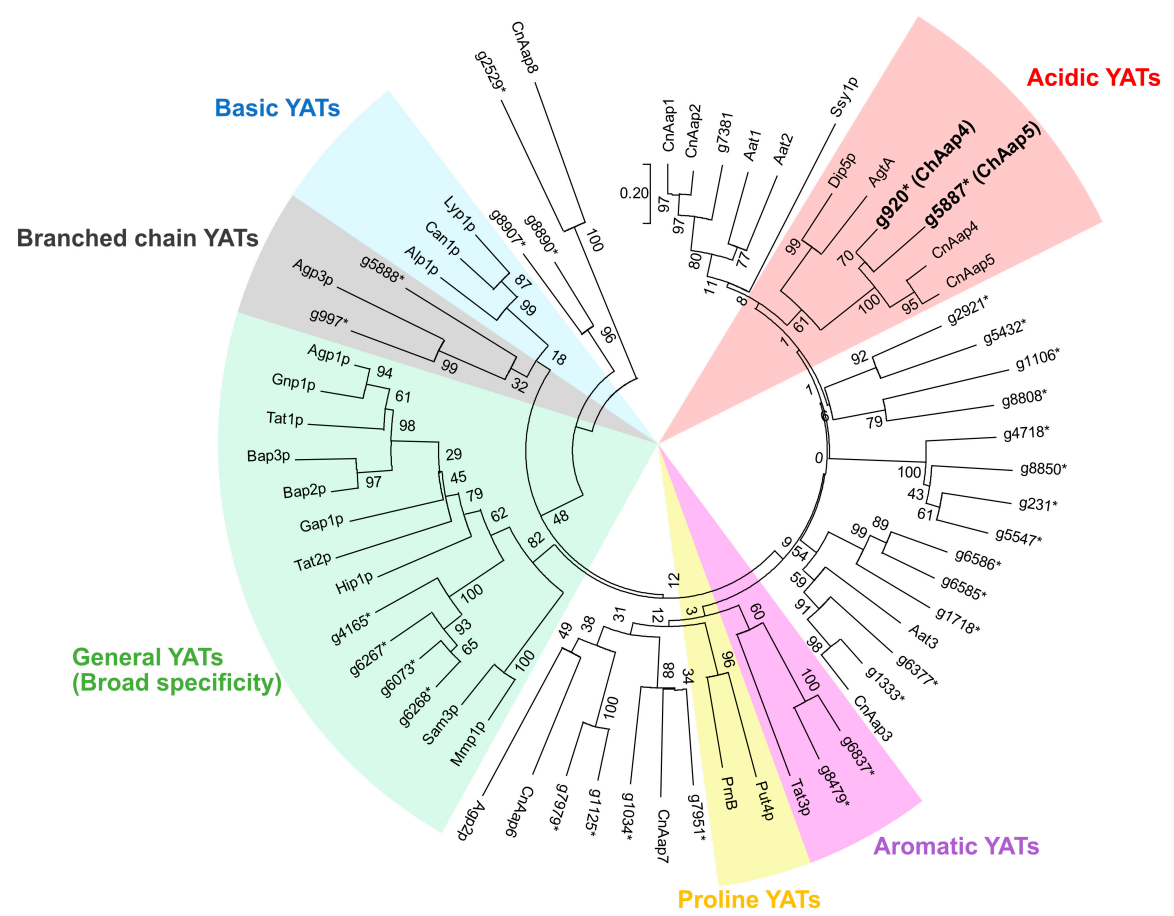

Figure 2. Phylogenetic relationship of Aaps homologs of C. humicola strain UJ1 and YATs. The phylogenetic tree was constructed by the neighbor-joining method with 1000 bootstrap trials using MEGA version 7.0. The numbers at nodes indicate bootstrap value as percentages. The asterisks indicate Aap homologs of strain UJ1. Accession numbers of the amino acid sequences used for the analysis were as follows: C. neoformans CnAap1 (CNAG_02539), CnAap2 (CNAG_07902), CnAap3 (CNAG_1118), CnAap4 (CNAG_00597), CnAap5 (CNAG_07367), CnAap6 (CNAG_07449), CnAap7 (CNAG_05345), and CnAap8 (CNAG_00574); Saccharomyces cerevisiae Tat1p (Uniprot: P38085), Tat2p (P38967), Tat3p (A4UZ28), Gap1p (P19145), Hip1p (P06775), Gnp1p (P48813), Agp1p (P25376), Agp2p (P38090), Agp3p (P43548), Bap2p (P38084), Bap3p (P41815), Sam3p (Q08986), Mmp1p (Q12372), Lyp1p (P32487), Alp1p (P38971), Can1p (P04817), Dip5p (P53388), Put4p (P15380), and Ssy1p (Q03770); Aspergillus nidulans AgtA (B2M1L6) and PrnB (P18696); Uromyces fabae Aat1 (Q96TU9), Aat2 (O00062), and Aat3 (Q700T6).

\section{TM1}

\begin{tabular}{|c|c|c|c|c|}
\hline AdiC & 10 & VGLIPVTLMVSGNIM GSG & VFLLPANLA-ST & 38 \\
\hline ChAap4 & 56 & LKPRQISMIAIGGAIGTG & LVIGSGTSLIRS & 85 \\
\hline ChAap5 & 47 & LKPRQISMIAIGGAIGAG & FLIGSGTSLARS & 76 \\
\hline Dip5p & 86 & LKARHISMIAIGGSLGTG & LLIGTGTALLTG & 115 \\
\hline PcDip5 & 38 & LKARHISMIAIGGAIGTG & LVVGTGSAL-TA & 66 \\
\hline AgtA & 40 & LKARHITMIAIGGAIGTG & LIIGTGSALEKA & 69 \\
\hline & \multicolumn{4}{|c|}{ TM6 } \\
\hline AdiC & 190 & FGAIQSTLNVTL WSFIGVE & SSASVAAGVVKN & 219 \\
\hline ChAap4 & 250 & FLGFVNALILAL FAYMGTE & ELVGVTVGEAKN & 279 \\
\hline ChAap5 & 240 & FLGFVNALVLAL LAFMGSE & LIGMTVGEAKN & 269 \\
\hline Dip5p & 283 & FVSFVAVFVYSL FSYTGIE & LTGIVCSEAEN & 312 \\
\hline PcDip5 & 235 & FLAFWSTMVQAT FAFLGTE & LIGVTVGEAQN & 264 \\
\hline AgtA & 242 & FYAFWATLVSAT FAYLGTE & LLVGVTVGEAQN & 272 \\
\hline
\end{tabular}

Figure 3. Comparison of amino acid sequences at transmembrane (TM) region 1 and 6 of ChAap4 and ChAap5 with E. coli AdiC (UniProKB: P60061) and three yeast dicarboxylic amino acid permeases: Dip5p (S. cerevisiae), PcDip5 (P. chrysogenum), and AgtA (A. nidulans). Transmembrane (TM) regions were predicted by Phobius (http:/ / phobius.sbc.su.se/). GXG and (F/Y)(S/A/T)(F/Y)XGXE motifs (where $\mathrm{X}$ is any amino acid) are boxed in red and blue, respectively. 


\subsection{Growth Characteristics of Chaap4 and Chaap5 Strains on Amino Acids}

To investigate the involvement of ChAap4 and ChAap5 in acidic amino acid uptake, we created strains with disruption of each gene (Chaap4 and Chaap5, Figure 1) and analyzed their growth on various amino acids at $\mathrm{pH} 7$ (Table 1). The gene disruptions were not lethal, and they did not result in growth suppression in medium containing glucose as the sole carbon source and $\mathrm{NH}_{4} \mathrm{Cl}$ as the sole nitrogen source, suggesting that the genes might not be necessary for normal cell growth. In addition, the gene-disrupted strains grew on aliphatic L-amino acids, L-methionine, L-serine, and L-dicarboxylic amino acid amides (L-Asn and L-Gln) as the sole nitrogen source, similarly as the wild-type strain. However, Chaap4 grew more slowly than the wild-type strain on acidic amino acids and L-Phe, and Chaap5 grew more slowly on L-Lys and L-Phe (Table 1). These results suggested the involvement of ChAap4 in the uptake of acidic amino acids and L-Phe, whereas ChAap5 appears to be involved in the uptake of L-Lys and L-Phe, suggesting that ChAap4 functions as an acidic Aap.

Table 1. Growth of Chaap4 and Chaap5 strains on various amino acids.

\begin{tabular}{|c|c|c|c|}
\hline & N Source & Chaap4 & Chaap5 \\
\hline \multirow{6}{*}{ Aliphatic } & None & Below Detection & Below Detection \\
\hline & $\mathrm{NH}_{4} \mathrm{Cl}$ & $95 \%$ & $98 \%$ \\
\hline & L-Ala & $106 \%$ & $115 \%$ \\
\hline & Gly & $108 \%$ & $115 \%$ \\
\hline & L-Ile & $95 \%$ & $107 \%$ \\
\hline & L-Val & $113 \%$ & $104 \%$ \\
\hline Aromatic & L-Phe & $60 \%$ * & $79 \%$ * \\
\hline Sulfur & L-Met & $102 \%$ & $95 \%$ \\
\hline Hydroxylated & L-Ser & $106 \%$ & $106 \%$ \\
\hline \multirow[t]{3}{*}{ Acidic } & L-Asp & $36 \% * * *$ & $94 \%$ \\
\hline & D-Asp & $67 \% * *$ & $102 \%$ \\
\hline & L-Glu & $84 \%$ ** & $103 \%$ \\
\hline \multirow[t]{2}{*}{ Amide } & L-Asn & $96 \%$ & $107 \%$ \\
\hline & L-Gln & $102 \%$ & $108 \%$ \\
\hline Basic & L-Lys & $96 \%$ & $92 \%$ * \\
\hline
\end{tabular}

The gene-disrupted strains were cultivated at $30{ }^{\circ} \mathrm{C}$ at $\mathrm{pH} 7.0$ in a synthetic media containing $2 \%(w / v)$ glucose and $10 \mathrm{mM}$ each amino acid as the sole nitrogen source and their growth at $\mathrm{OD}_{600}$ are expressed as percentages of that of the wild-type strain in mid-logarithmic phase. Statistical differences were ascertained by Student's $t$-test, ${ }^{*} p<0.05,{ }^{* *} p<0.01$, and ${ }^{* *} p<0.001$ ( $n=3$ for each strain).

\subsection{Effect of Medium $p H$ on the Growth of Chaap4 and Chaap5 Strains on Amino Acids}

Acidic YATs are suggested to more tightly bind to the unprotonated forms of acidic amino acids [36-38], and substrate transport by AdiC and GadC (a Glu/ $\gamma$-aminobutyrate antiporter of E. coli) is pH-dependent [39]. We, therefore, investigated the effect of culture medium $\mathrm{pH}$ on the growth of Chaap 4 and Chaap 5 strains on amino acids as the sole nitrogen source (Figure 4). The gene-disrupted strains and wild-type strain displayed similar growth on $\mathrm{NH}_{4} \mathrm{Cl}$ as the sole nitrogen source at all pHs tested (Figure $4 \mathrm{~A}-\mathrm{C}$ ). Contrarily, although the Chaap4 strain grew similarly well as the wild-type strain on L-Asp, D-Asp, and L-Glu at pH 3.0 (Figure 4D,G,J), its growth on L-Asp, D-Asp, and L-Glu was inhibited or completely abolished at pH 10 (Figure 4F,I,L). In addition, on L-Phe, both gene-disrupted strains grew more slowly than the wild-type stain at all $\mathrm{pHs}$ tested (Figure $4 \mathrm{M}, \mathrm{N}, \mathrm{O}$ ), and the growth of Chaap 4 was more strongly repressed at higher $\mathrm{pHs}$, as observed on acidic amino acids. These results suggested that ChAap4 might function as an acidic Aap and play a critical role in growth on both L-Asp and D-Asp under high alkaline conditions. 

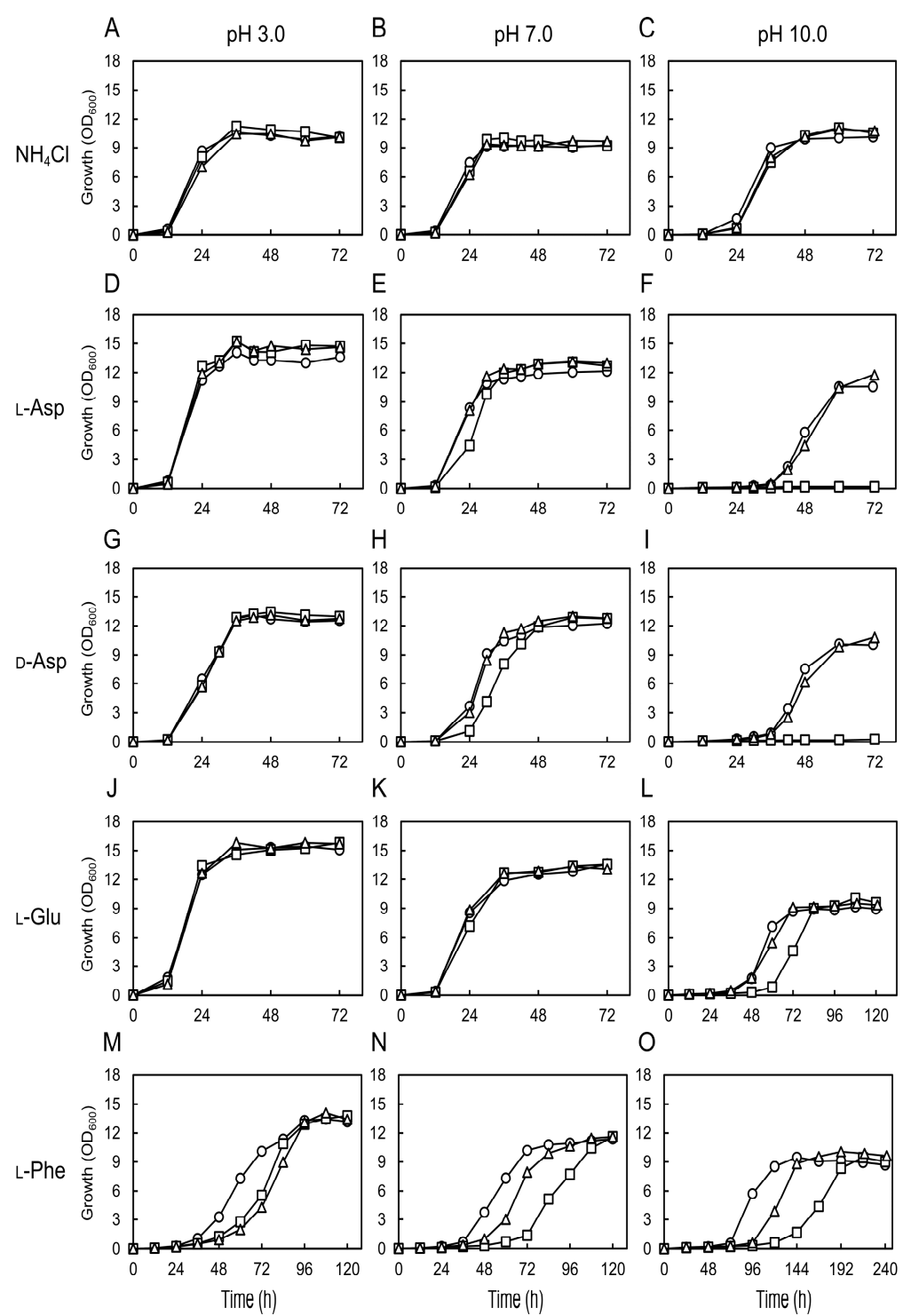

Figure 4. Effect of medium $\mathrm{pH}$ on the growth of Chaap strains on amino acids. The wild-type (strain UJ1) (open circles), Chaap4 (open squares), and Chaap5 (open triangles) strains were cultivated at $30{ }^{\circ} \mathrm{C}$ in a medium containing $10 \mathrm{mM} \mathrm{NH}_{4} \mathrm{Cl}$ or each amino acid as the sole nitrogen source. Initial $\mathrm{pH}$ of the media was adjusted to $3.0(\mathbf{A}, \mathbf{D}, \mathbf{G}, \mathbf{J}, \mathbf{M}), 7.0(\mathbf{B}, \mathbf{E}, \mathbf{H}, \mathbf{K}, \mathbf{N})$, or $10.0(\mathbf{C}, \mathbf{F}, \mathbf{I}, \mathbf{L}, \mathbf{O})$.

As deletion of ChAAP4 abolished its growth on D-Asp and L-Asp at pH 10 (Figure $4 \mathrm{~F}, \mathrm{I})$, to clarify the relationship between the function of ChAap4 and ChAap5 and the expression of ChDDO, we analyzed DDO activity and ChDDO transcription in the Chaap4 and Chaap5 strains in the presence or absence of D-Asp as the sole nitrogen and carbon sources at different $\mathrm{pHs}$ (Figure 5). Both DDO activity and ChDDO transcription were only induced in the presence of D-Asp but not L-Asp and absence of any amino acids. ChDDO expression profile was similar between the wild-type and Chaap 5 strains at all $\mathrm{pHs}$ tested, and they gradually decreased with increasing $\mathrm{pH}$. Conversely, the induction level of DDO activity and the transcription by D-Asp were markedly lower in the Chaap4 strain than in the wild-type strain at all pHs, and no activity was detected at $\mathrm{pH} 10$ (Figure 5). These results suggested that the uptake of D-Asp by ChAap4 might participate in the induction of ChDDO expression by D-Asp. 

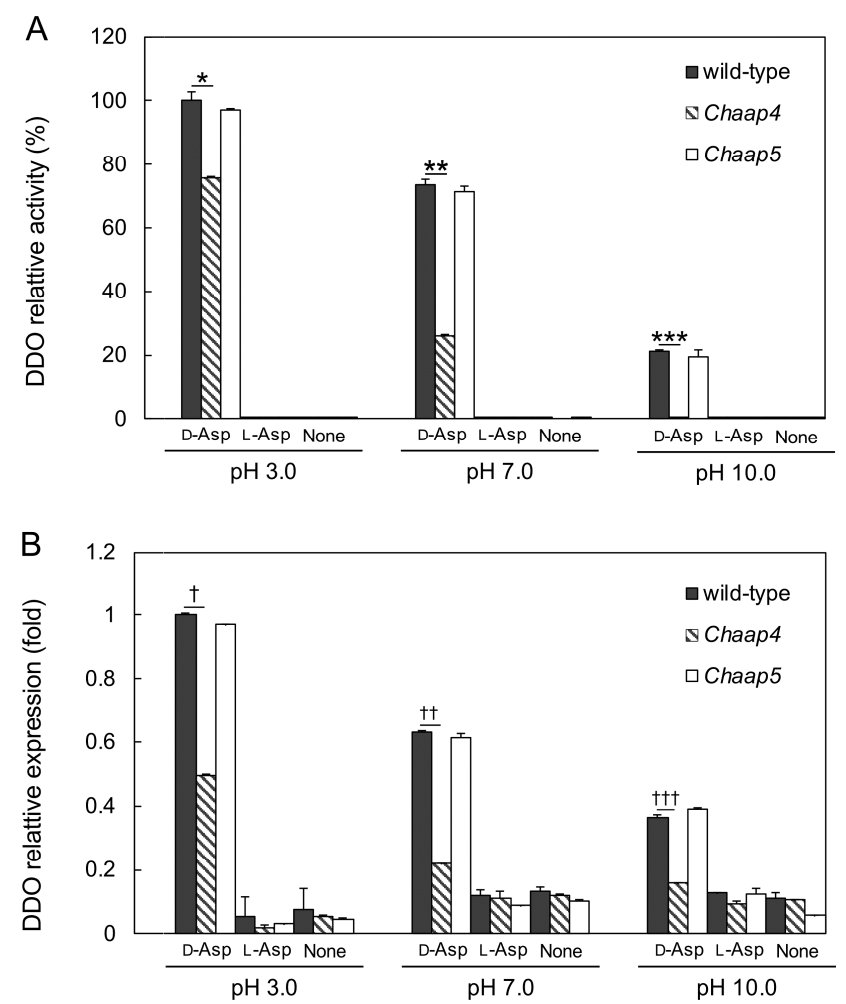

Figure 5. The expression of ChDDO gene in Chaap strains grown on D-Asp at different pHs. (A) DDO activity in the extracts from the cells grown in a synthetic medium containing (D-Asp or L-Asp) or not containing (None). For this, $60 \mathrm{mM}$ D-Asp or L-Asp was used as the sole nitrogen and carbon sources at different $\mathrm{pHs}$ at $30{ }^{\circ} \mathrm{C}$. The enzyme activity is expressed as a percentage of that of the wild-type strain at $\mathrm{pH}$ 3.0. Statistical differences were ascertained by Student's $t$-test, ${ }^{*} p<5 \times 10^{-4}$, ** $p<5 \times 10^{-6}$, and ${ }^{* *} p<1 \times 10^{-6}$. (B) Transcription of ChDDO gene. The gene transcription was analyzed by qRT-PCR using total RNA from cells grown under the same conditions as in the analysis of DDO activity, normalized to TAF10 gene transcription, and expressed as a relative ratio of that of the wild-type strain at $\mathrm{pH}$ 3.0. Statistical differences were ascertained by Welch's $t$-test, $+p<5 \times 10^{-4}$, $++p<5 \times 10^{-7}$, and $+++p<1 \times 10^{-7}$. The values are the means of three independent experiments, and the error bars are the standard deviations.

\section{Discussion}

Aaps that transport similar amino acids are usually classified into the same clade in a phylogenetic tree (Figure 2) [40]. In Ascomycota fungi such as S. cerevisiae (Dip5p) and A. nidulans (AgtA), one Aap is usually classified into the acidic Aap clade. Conversely, two Aaps were classified into the acidic Aap clade in C. humicola (ChAap4 and ChAap5) and C. neoformans (CnAap4 and CnAap5). In this study, one of the two Aaps of C. humicola, ChAap4, was suggested to mediate the uptake of acidic amino acids, whereas ChAap5 did not mediate their uptake (Figure 4). Similar to the results of the present study, CnAap4 of an opportunistic pathogen C. neoformans is suggested to transport L-Asp, whereas CnAap5 does not transport this amino acid at the growth temperature of $30^{\circ} \mathrm{C}$, although the enzyme is suggested to transport L-Asp at the growth temperature of $37^{\circ} \mathrm{C}$ [41]. The two Aaps in each Basidiomycota fungus share high amino acid sequence identity, and the exon-intron structures of their coding genes are identical (Figure S2), suggesting that the genes might have been generated evolutionarily via gene duplication. The gene duplication and the different substrate specificities of the two Aaps might occur to enable the utilization of various amino acids for cell growth. Inconsistency between the substrate specificity and classification in the phylogenetic tree is also found in Aaps of S. cerevisiae: The histidine permease Hip1p and tryptophan/tyrosine permease Tat2p are classified into the same clade as the broad substrate specificity permease Gap1p (Figure 2) [17]. As they reported, 
coclustering in the same clade is not always sufficient to predict the substrate specificity of YATs. It is possible that the existence of multiple Aaps in the same clade indicates that they have different substrate specificities.

The substrate recognition mode in Aaps has been well described in the bacterial L-Arg permease AdiC [35,42]. In the crystal structure of AdiC, the positively charged L-Arg is placed in an occluded chamber with negative surface potential (Figure 6A). This electrostatic interaction is therefore considered to be one contributor to substrate specificity and transport [35]. The electrostatic potential in the substrate-binding site of ChAap4 model structure was suggested to be positive, whereas that of ChAap5 appeared to be more neutral (Figure $6 \mathrm{~B}, \mathrm{C}$ ). These findings suggested that the electrostatic potential of ChAap4 might contribute to the substrate preference toward L-Asp and the different substrate preference compared with ChAap5. In addition to the electrostatic interaction, Trp293 in TM8 and Asn101 in TM3 in AdiC are suggested to be involved in the substrate preference by interacting with the guanidinium group of the L-Arg substrate through a $\pi$-cation interaction and a hydrogen bond, respectively (Figure 7A) [42]. In ChAap4 model structure, Tyr145 and Ile148 in TM3 were located at the spatially corresponding positions, respectively (Figure 7B). Similar to the amino acid residues of AdiC, these residues might contribute to the substrate preference toward L-Phe of ChAap4 through a $\pi-\pi$ interaction and a hydrophobic interaction, respectively. However, it should be noted that in addition to the substrate-binding site, other regions are also known to affect the substrate specificity of Aaps [43]. Further studies, such as mutational studies, are needed to clearly demonstrate the structural factors involved in the substrate specificity of ChAap4.

A

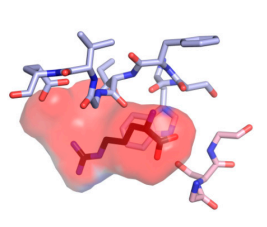

B

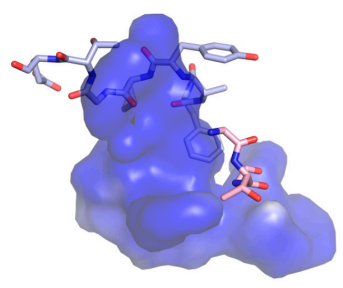

C

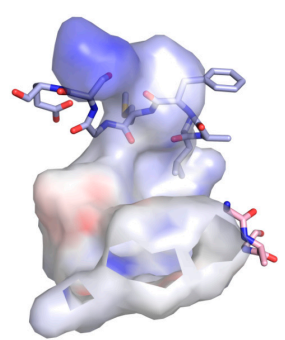

Figure 6. Electrostatic potential of substrate-binding pockets of AdiC (A), ChAap4 (B), and ChAap5 (C). The electrostatic potential was calculated using the software PyMOL 2.3x and is showed by a gradient of blue (positive charge) and red (negative charge) colors. The carbon atoms of L-Arg in AdiC is colored in black. Oxygen and nitrogen atoms are shown in red and blue, respectively. GXG and $(\mathrm{F} / \mathrm{Y})(\mathrm{S} / \mathrm{A} / \mathrm{T})(\mathrm{F} / \mathrm{Y}) \mathrm{XGXE}$ motifs are displayed in light pink and light blue, respectively.

A

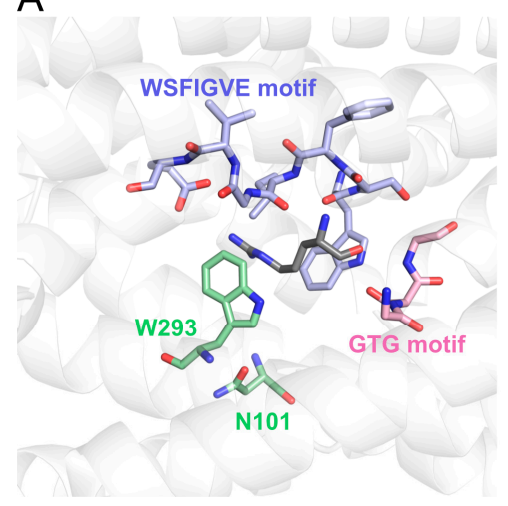

B

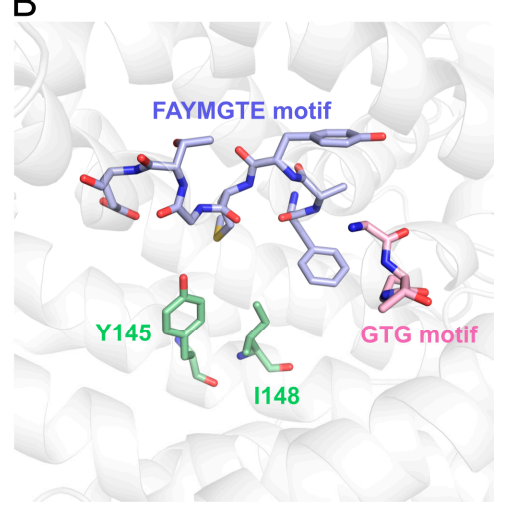

Figure 7. Substrate-binding site of AdiC (A) and ChAap4 (B). Trp293 in TM8 and Asn101 in TM3 in AdiC and Tyr145 and Ile148 in TM3 in ChAap4 potentially involved in the substrate preference are displayed in light green. 
Fungal Aaps are proton symporters that can transport amino acids using proton gradient (outside > inside cell) as a driving force $[19,20]$. Therefore, the decreased growth rate on amino acids with increasing $\mathrm{pH}$, observed in this study (Figure 4), might be due to the decreased amino-acid uptake rate with increasing $\mathrm{pH}$, which might cause the significant decrease in the induction of ChDDO expression by D-Asp (Figure 5). In fungi, acidic amino acids are transported into cells by both general (nonspecific) and acidic Aaps [36-38,43]. It has been reported that the uptake of L-Asp by the general Aap Gap1p decreases with increasing $\mathrm{pH}$ [44], and the general amino acid transport system of the fungus P. chrysogenum preferentially transports the uncharged forms of L-Glu over the charged forms [37]. On the contrary, the acidic amino acid transporter system of P. chrysogenum is suggested to accept both the uncharged and mononegative forms with equal affinities [37]. Together with these findings, the significant growth of Chaap4 strain (ChAAP4 gene-disrupted strain) on D- and $\mathrm{L}-\mathrm{Asp}$ at acidic and neutral $\mathrm{pH}$ conditions suggested that unidentified general Aaps as well as ChAap4 might catalyze the uptake of D- and L-Asp at the $\mathrm{pH}$ conditions in C. humicola (Figure 4D-I), whereas ChAap4 might play a major role in the acidic amino acid uptake at high alkaline conditions. Additionally, the significant growth of the Chaap4 strain on L-Glu at an alkaline $\mathrm{pH}$ implied the presence of a Glu-specific acidic Aap. Alternatively, there is a possibility that a dicarboxylic permease might be involved in L-Glu uptake at higher alkaline $\mathrm{pH}$ [45], because approximately $70 \%$ of L-Glu is estimated to be present as the dianionic form based on $\mathrm{pKa}=9.63$ of the $\alpha$-amino group of $\mathrm{L}-\mathrm{Glu}$ at $\mathrm{pH} 10$. The similar growth phenotype of Chaap4 strain on D- and L-Asp (Figure 4D-I) and the findings that Gap1p can transport some D-amino acids as well as various L-amino acids [21], D- and $\mathrm{L}$-amino acids are suggested to be transported by a common transport system in S. cerevisiae [46], and Neurospora crassa possesses an active transporter system for D- and L-acidic amino acids [36] suggesting that ChAap4 could transport both Asp enantiomers.

The transcription of the fungal genes involved in the utilization of amino acids is regulated by various regulatory systems, including nitrogen catabolite repression [47], the SPS sensor system [48], the TOR regulatory pathway [49], and the GAAC pathway [50], by sensing amino acids inside or outside cells. The complicated and sophisticated network creates a broad regulatory system that can regulate the precise control of gene expression. Meanwhile, transcription of PUT1 and PUT2, which encode the proline utilization enzymes proline oxidase and $\Delta^{1}$-pyrroline-5-carboxylate dehydrogenase, respectively, is specifically induced only by the presence of L-Pro inside cells. Specifically, L-Pro binds to the transcriptional activator Put3p, which binds to the DNA upstream of the target genes and significantly induces gene transcription [51]. Similarly, as ChDDO transcription is specifically induced only in the presence of D-Asp and not subjected to nitrogen catabolite repression [7,8], a D-Asp-specific transcriptional activation system is likely to exist in C. humicola strain UJ1. A significant relationship between the growth of C. humicola on D-Asp and the transcription of ChDDO suggested that D-Asp inside the cells might be recognized by an unknown D-Asp-specific sensor protein or transcriptional activator protein that can induce the transcription.

\section{Conclusions}

In conclusion, we propose the following model for the relationship between the inducible expression of ChDDO in the presence of D-Asp and the uptake of D-Asp by ChAap4 and other Aaps in C. humicola strain UJ1 (Figure 8). In acidic and neutral environments, D-Asp uptake might be mediated by multiple Aaps, including ChAap4 and Gap(s). The DAsp uptake activity of Gap(s) might be gradually decreased by changing the environmental $\mathrm{pH}$ from neutral to alkaline conditions and completely abolished under highly alkaline conditions, whereas ChAap4 could still import D-Asp even under high alkalinity. D-Asp imported by the Aaps might induce the transcription of ChDDO to utilize D-Asp for cell growth and reduce D-Asp-mediated toxicity. At present, the sensor that recognizes D-Asp inside cells and induces gene transcription is unknown, and future research should aim to identify the involved protein(s) to clarify the entire mechanism of the D-Asp-specific 
gene transcriptional activation. The clarification of the whole regulation mechanism of $D D O$ gene expression in the yeast might contribute to the further understanding of the physiological functions of DDO and D-Asp in not only fungi but also animals including human, and, furthermore, the onset mechanism of the psychiatric disease schizophrenia suggested to be associated with DDO and D-Asp.

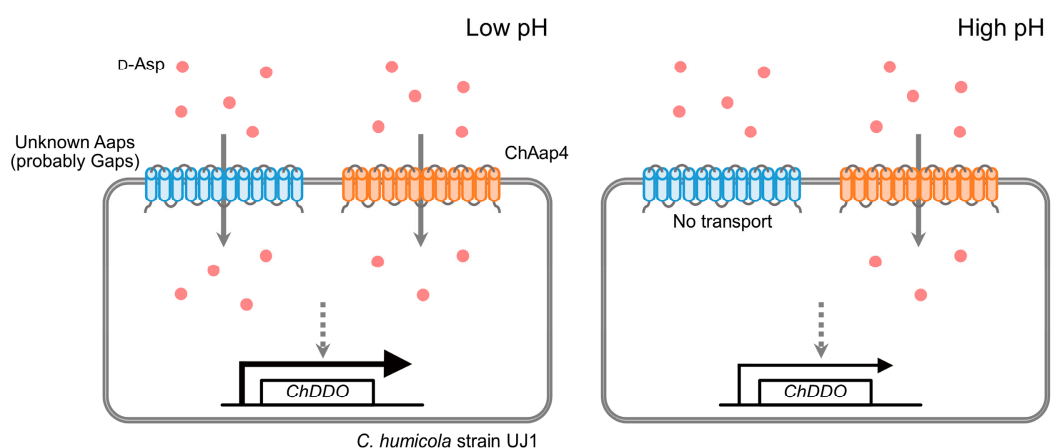

Figure 8. Relationship between D-Asp uptake by ChAap4 and ChDDO gene expression in the yeast C. humicola strain UJ1. In acidic and neutral environments, D-Asp molecules (red circles) are transported (grey arrows) by ChAap4 (red proteins) and unknown Aaps (probably Gaps, blue proteins). In high alkaline environments, the D-Asp uptake by Gaps is abolished but not by ChAap4. Intracellular D-Asp induces ChDDO gene expression via an unknown signaling pathway (grey dotted arrows).

Supplementary Materials: The following are available online at https:/ /www.mdpi.com/2076-260 7/9/1/192/s1, Figure S1: Comparison of amino acid sequences of ChAap4 and ChAap5 with E. coli AdiC (UniProKB: P60061) and three yeast dicarboxylic amino acid permeases: Dip5p (S. cerevisiae), PcDip5 (P. chrysogenum), and AgtA (A. nidulans). Figure S2: Exon-intron structures of ChAAP4, ChAAP5, and acidic Aap genes. Table S1: Primers used in this study.

Author Contributions: Conceptualization, D.I. and S.T.; methodology, D.I. and S.T.; validation, D.I. and S.T.; formal analysis, D.I.; investigation, D.I.; resources, Y.K. and S.T.; data curation, D.I.; writingoriginal draft preparation, D.I. and S.T.; writing—review and editing, D.I. and S.T.; visualization, D.I.; supervision, S.T.; project administration, S.T., funding acquisition, S.T. and Y.K. All authors have read and agreed to the published version of the manuscript.

Funding: This research received no external funding.

Institutional Review Board Statement: Not applicable.

Informed Consent Statement: Not applicable.

Data Availability Statement: The data presented in this study are available on request from the corresponding author.

Conflicts of Interest: The authors declare no conflict of interest.

\section{References}

1. D'Aniello, A.; Palescandolo, R.; Scardi, B. The distribution of the D-aspartate oxidase activity in Cephalopoda. Comp. Biochem. Physiol. B 1975, 50, 209-210. [CrossRef]

2. Yamada, R.; Nagasaki, H.; Wakabayashi, Y.; Iwashima, A. Presence of D-aspartate oxidase in rat liver and mouse tissues. Biochim. Biophys. Acta 1988, 965, 202-205. [CrossRef]

3. Kera, Y.; Nagasaki, H.; Iwashima, A.; Yamada, R. Presence of D-aspartate oxidase and free D-aspartate in amphibian (Xenopus laevis, Cynops pyrrhogaster) tissues. Comp. Biochem. Physiol. B 1992, 103, 345-348. [CrossRef]

4. Kera, Y.; Aoyama, H.; Watanabe, N.; Yamada, R.H. Distribution of D-aspartate oxidase and free D-glutamate and D-aspartate in chicken and pigeon tissues. Comp. Biochem. Physiol. B Biochem. Mol. Biol. 1996, 115, 121-126. [CrossRef]

5. Yamada, R.; Ujiie, H.; Kera, Y.; Nakase, T.; Kitagawa, K.; Imasaka, T.; Arimoto, K.; Takahashi, M.; Matsumura, Y. Purification and properties of D-aspartate oxidase from Cryptococcus humicolus UJ1. Biochim. Biophys. Acta 1996, 1294, 153-158. [CrossRef] 
6. Takahashi, S. D-Aspartate Oxidase: Distribution, Functions, Properties, and Biotechnological Applications. Appl. Microbiol. Biotechnol. 2020, 104, 2883-2895. [CrossRef] [PubMed]

7. Takahashi, S.; Takahashi, T.; Kera, Y.; Matsunaga, R.; Shibuya, H.; Yamada, R.H. Cloning and expression in Escherichia coli of the D-aspartate oxidase gene from the yeast Cryptococcus humicola and characterization of the recombinant enzyme. J. Biochem. 2004, 135, 533-540. [CrossRef]

8. Takahashi, S.; Kakuichi, T.; Fujii, K.; Kera, Y.; Yamada, R.H. Physiological role of D-aspartate oxidase in the assimilation and detoxification of D-aspartate in the yeast Cryptococcus humicola. Yeast 2005, 22, 1203-1212. [CrossRef]

9. D'Aniello, A.; D’Onofrio, G.; Pischetola, M.; D'Aniello, G.; Vetere, A.; Petrucelli, L.; Fisher, G.H. Biological role of D-amino acid oxidase and D-aspartate oxidase. Effects of D-amino acids. J. Biol. Chem. 1993, 268, 26941-26949. [CrossRef]

10. Huang, A.S.; Beigneux, A.; Weil, Z.M.; Kim, P.M.; Molliver, M.E.; Blackshaw, S.; Nelson, R.J.; Young, S.G.; Snyder, S.H. D-Aspartate regulates melanocortin formation and function: Behavioral alterations in D-aspartate oxidase-deficient mice. J. Neurosci. 2006, 26, 2814-2819. [CrossRef]

11. Errico, F.; Nuzzo, T.; Carella, M.; Bertolino, A.; Usiello, A. The Emerging Role of Altered D-Aspartate Metabolism in Schizophrenia: New Insights from Preclinical Models and Human Studies. Front. Psychiatry 2018, 9, 559. [CrossRef] [PubMed]

12. Simonetta, M.P.; Verga, R.; Fretta, A.; Hanozet, G.M. Induction of D-amino-acid oxidase by D-alanine in Rhodotorula gracilis grown in defined medium. Microbiology 1989, 135, 593-600. [CrossRef]

13. Godard, P.; Urrestarazu, A.; Vissers, S.; Kontos, K.; Bontempi, G.; van Helden, J.; Andre, B. Effect of 21 different nitrogen sources on global gene expression in the yeast Saccharomyces cerevisiae. Mol. Cell. Biol. 2007, 27, 3065-3086. [CrossRef] [PubMed]

14. Wakayama, M.; Nakashima, S.; Sakai, K.; Moriguchi, M. Isolation, Enzyme-Production and Characterization of D-Aspartate Oxidase from Fusarium-Sacchari var. Elongatum Y-105. J. Ferment. Bioeng. 1994, 78, 377-379. [CrossRef]

15. Fukunaga, S.; Yuno, S.; Takahashi, M.; Taguchi, S.; Kera, Y.; Odani, S.; Yamada, R.H. Purification and properties of D-glutamate oxidase from Candida boidinii 2201. J. Ferment. Bioeng. 1998, 85, 579-583. [CrossRef]

16. Yamada, R.; Nagasaki, H.; Nagata, Y.; Wakabayashi, Y.; Iwashima, A. Administration of D-aspartate increases D-aspartate oxidase activity in mouse liver. Biochim. Biophys. Acta 1989, 990, 325-328. [CrossRef]

17. Gournas, C.; Athanasopoulos, A.; Sophianopoulou, V. On the Evolution of Specificity in Members of the Yeast Amino Acid Transporter Family as Parts of Specific Metabolic Pathways. Int. J. Mol. Sci. 2018, 19, 1398. [CrossRef]

18. Reizer, J.; Finley, K.; Kakuda, D.; MacLeod, C.L.; Reizer, A.; Saier, M.H., Jr. Mammalian integral membrane receptors are homologous to facilitators and antiporters of yeast, fungi, and eubacteria. Protein Sci. 1993, 2, 20-30. [CrossRef]

19. Horak, J. Amino acid transport in eucaryotic microorganisms. Biochim. Biophys. Acta 1986, 864, 223-256. [CrossRef]

20. Horak, J. Yeast nutrient transporters. Biochim. Biophys. Acta 1997, 1331, 41-79. [CrossRef]

21. Grenson, M.; Hou, C.; Crabeel, M. Multiplicity of the amino acid permeases in Saccharomyces cerevisiae. IV. Evidence for a general amino acid permease. J. Bacteriol. 1970, 103, 770-777. [CrossRef] [PubMed]

22. Jauniaux, J.C.; Grenson, M. GAP1, the general amino acid permease gene of Saccharomyces cerevisiae. Nucleotide sequence, protein similarity with the other bakers yeast amino acid permeases, and nitrogen catabolite repression. Eur. J. Biochem. 1990, 190, 39-44. [CrossRef] [PubMed]

23. Andre, B. An overview of membrane transport proteins in Saccharomyces cerevisiae. Yeast 1995, 11, 1575-1611. [CrossRef] [PubMed]

24. Regenberg, B.; Holmberg, S.; Olsen, L.D.; Kielland-Brandt, M.C. Dip5p mediates high-affinity and high-capacity transport of L-glutamate and L-aspartate in Saccharomyces cerevisiae. Curr. Genet. 1998, 33, 171-177. [CrossRef] [PubMed]

25. Regenberg, B.; During-Olsen, L.; Kielland-Brandt, M.C.; Holmberg, S. Substrate specificity and gene expression of the amino-acid permeases in Saccharomyces cerevisiae. Curr. Genet. 1999, 36, 317-328. [CrossRef]

26. Trip, H.; Evers, M.E.; Kiel, J.A.; Driessen, A.J. Uptake of the beta-lactam precursor alpha-aminoadipic acid in Penicillium chrysogenum is mediated by the acidic and the general amino acid permease. Appl. Environ. Microbiol. 2004, 70, 4775-4783. [CrossRef]

27. Apostolaki, A.; Erpapazoglou, Z.; Harispe, L.; Billini, M.; Kafasla, P.; Kizis, D.; Penalva, M.A.; Scazzocchio, C.; Sophianopoulou, V. AgtA, the dicarboxylic amino acid transporter of Aspergillus nidulans, is concertedly down-regulated by exquisite sensitivity to nitrogen metabolite repression and ammonium-elicited endocytosis. Eukaryot. Cell 2009, 8, 339-352. [CrossRef]

28. Takahashi, S.; Matsunaga, R.; Kera, Y.; Yamada, R.H. Isolation of the Cryptococcus humicolus URA3 gene encoding orotidine-5'phosphate decarboxylase and its use as a selective marker for transformation. J. Biosci. Bioeng. 2003, 96, 23-31. [CrossRef]

29. Imanishi, D.; Abe, K.; Kera, Y.; Takahashi, S. Draft Genome Sequence of the Yeast Vanrija humicola (Formerly Cryptococcus humicola) Strain UJ1, a Producer of D-Aspartate Oxidase. Genome Announc. 2018, 6, e00068-18. [CrossRef]

30. Altschul, S.F.; Gish, W.; Miller, W.; Myers, E.W.; Lipman, D.J. Basic local alignment search tool. J. Mol. Biol. 1990, 215 , 403-410. [CrossRef]

31. Fernandes, J.D.; Martho, K.; Tofik, V.; Vallim, M.A.; Pascon, R.C. The Role of Amino Acid Permeases and Tryptophan Biosynthesis in Cryptococcus neoformans Survival. PLoS ONE 2015, 10, e0132369. [CrossRef] [PubMed]

32. Kumar, S.; Stecher, G.; Tamura, K. MEGA7: Molecular Evolutionary Genetics Analysis Version 7.0 for Bigger Datasets. Mol. Biol. Evol. 2016, 33, 1870-1874. [CrossRef] [PubMed]

33. Molla, G.; Piubelli, L.; Volonte, F.; Pilone, M.S. Enzymatic detection of D-amino acids. Methods Mol. Biol. 2012, 794, 273-289. [CrossRef] [PubMed] 
34. Livak, K.J.; Schmittgen, T.D. Analysis of relative gene expression data using real-time quantitative PCR and the $2^{-\Delta \Delta C}{ }_{T}$ Method. Methods 2001, 25, 402-408. [CrossRef] [PubMed]

35. Gao, X.; Zhou, L.; Jiao, X.; Lu, F.; Yan, C.; Zeng, X.; Wang, J.; Shi, Y. Mechanism of substrate recognition and transport by an amino acid antiporter. Nature 2010, 463, 828-832. [CrossRef]

36. Pall, M.L. Amino acid transport in Neurospora crassa. 3. Acidic amino acid transport. Biochim. Biophys. Acta 1970, 211, 513-520. [CrossRef]

37. Hunter, D.R.; Segel, I.H. Acidic and basic amino acid transport systems of Penicillium chrysogenum. Arch. Biochem. Biophys. 1971, 144, 168-183. [CrossRef]

38. Robinson, J.H.; Anthony, C.; Drabble, W.T. The acidic amino-acid permease of Aspergillus nidulans. J. Gen. Microbiol. 1973, 79, 53-63. [CrossRef]

39. Ma, D.; Lu, P.; Yan, C.; Fan, C.; Yin, P.; Wang, J.; Shi, Y. Structure and mechanism of a glutamate-GABA antiporter. Nature 2012, 483, 632-636. [CrossRef]

40. De Vries, R.P.; Riley, R.; Wiebenga, A.; Aguilar-Osorio, G.; Amillis, S.; Uchima, C.A.; Anderluh, G.; Asadollahi, M.; Askin, M.; Barry, K.; et al. Comparative genomics reveals high biological diversity and specific adaptations in the industrially and medically important fungal genus Aspergillus. Genome Biol. 2017, 18, 28. [CrossRef]

41. Martho, K.F.; de Melo, A.T.; Takahashi, J.P.; Guerra, J.M.; Santos, D.C.; Purisco, S.U.; Melhem, M.S.; Fazioli, R.D.; Phanord, C.; Sartorelli, P.; et al. Amino Acid Permeases and Virulence in Cryptococcus neoformans. PLoS ONE 2016, 11, e0163919. [CrossRef] [PubMed]

42. Kowalczyk, L.; Ratera, M.; Paladino, A.; Bartoccioni, P.; Errasti-Murugarren, E.; Valencia, E.; Portella, G.; Bial, S.; Zorzano, A.; Fita, I.; et al. Molecular basis of substrate-induced permeation by an amino acid antiporter. Proc. Natl. Acad. Sci. USA 2011, 108, 3935-3940. [CrossRef] [PubMed]

43. Gournas, C.; Prevost, M.; Krammer, E.M.; Andre, B. Function and Regulation of Fungal Amino Acid Transporters: Insights from Predicted Structure. Adv. Exp. Med. Biol. 2016, 892, 69-106. [CrossRef] [PubMed]

44. Olivera, H.; Gonzalez, A.; Pena, A. Regulation of the amino acid permeases in nitrogen-limited continuous cultures of the yeast Saccharomyces cerevisiae. Yeast 1993, 9, 1065-1073. [CrossRef]

45. Casal, M.; Paiva, S.; Queiros, O.; Soares-Silva, I. Transport of carboxylic acids in yeasts. FEMS Microbiol. Rev. 2008, 32, 974-994. [CrossRef]

46. Rytka, J. Positive selection of general amino acid permease mutants in Saccharomyces cerevisiae. J. Bacteriol. 1975, 121, 562-570. [CrossRef]

47. Hofman-Bang, J. Nitrogen catabolite repression in Saccharomyces cerevisiae. Mol. Biotechnol. 1999, 12, 35-73. [CrossRef]

48. Forsberg, H.; Ljungdahl, P.O. Genetic and biochemical analysis of the yeast plasma membrane Ssy1p-Ptr3p-Ssy5p sensor of extracellular amino acids. Mol. Cell. Biol. 2001, 21, 814-826. [CrossRef]

49. Conrad, M.; Schothorst, J.; Kankipati, H.N.; Van Zeebroeck, G.; Rubio-Texeira, M.; Thevelein, J.M. Nutrient sensing and signaling in the yeast Saccharomyces cerevisiae. FEMS Microbiol. Rev. 2014, 38, 254-299. [CrossRef]

50. Hinnebusch, A.G. Translational regulation of GCN4 and the general amino acid control of yeast. Annu. Rev. Microbiol. 2005, 59, 407-450. [CrossRef]

51. Sellick, C.A.; Reece, R.J. Modulation of transcription factor function by an amino acid: Activation of Put3p by proline. EMBO J. 2003, 22, 5147-5153. [CrossRef] [PubMed] 